\title{
北京市北运河沉积物对氮、磷的吸附/解吸动力学特征
}

\author{
胡洁蕴, 李淑芹**, 宋 歌, 刘寒青, 张 釒郎 琪 \\ (中国农业大学水利与土木工程学院, 北京 100083)
}

\begin{abstract}
摘 要: 选取北运河土沟、榆林庄及和合站 3 个典型河道断面, 进行河道 $0 \sim 60 \mathrm{~cm}$ 深度沉积物对氨氮、磷酸盐的吸附/解 吸批平衡静态试验研究, 采用多种动力学模型分析北运河沉积物对氮、磷的吸附/解吸动力学特性, 提出适用于北运河沉 积物吸附/解吸特性的动力学模型, 并对模型参数的主要影响因素进行探讨. 结果表明: 1) 北运河典型断面各深度的沉积 物对氨氮和磷酸盐的吸附/解吸过程总体呈现 3 个阶段: 快反应阶段一慢反应阶段一平衡阶段; 在 $0 \sim 0.5 \mathrm{~h}$ 的快反应阶段 可完成吸附或解吸总量的 $60 \%$, 且对氮、磷的吸附速率大于解吸速率. 2) 北运河各断面对氨氮与磷酸盐的平衡吸附量表

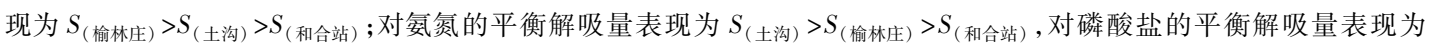
$S_{\text {(和合站) }}>S_{\text {(土沟 })}>S_{\text {(榆林庄) }}$, 沉积物对氨氮和磷酸盐的吸附以化学吸附为主, 平衡吸附与解吸量随断面深度的增加而减小, $0 \sim 20 \mathrm{~cm}$ 表层沉积物对氮、磷的吸附能力较强. 3) Lagergren 二级动力学模型对各深度沉积物的吸附/解吸过程拟合最优, 模型参数公式为 $k_{2}=S_{\text {max }}^{-0.369}+0.163 ; q_{\mathrm{e}}=0.022 S_{\text {max }}+18.077 K_{\mathrm{f}}+41.947$. 通过模拟得出在 $400 \mathrm{mg} / \mathrm{L}$ 氮、磷浓度下吸附 于沉积物中的氮、磷污染物会随着解吸过程释放 $52 \% \sim 80 \%$ 的氨氮和 $6 \% \sim 42 \%$ 的磷酸盐, 可能不仅二次污染上覆水体, 还 随河水下渗从而对地下水质形成潜在污染风险.
\end{abstract}

关键词: 北运河;沉积物;氮; 磷; 吸附;解吸;吸附速率

\section{Adsorption/desorption dynamic characteristic of sediments on nitrogen and phosphorus in the north Grand Canal of Beijing}

\author{
HU Jieyun, LI Shuqin ** , SONG Ge, LIU Hanqing, ZHANG Xin \& LANG Qi \\ (College of Water Resources \& Civil Engineering, China Agricultural University, Beijing 100083, P.R.China)
}

\begin{abstract}
Typical sections of north Grand Canal in Beijing ( Tugou, Yulin Village and Hehe Station) were selected, and the upper 0-60 cm sediments were collected to operate batch equilibrium static experiment on adsorption and desorption of nitrogen or phosphorus. Adsorption/desorption dynamic characteristic analysis was developed combing the experiment result and several dynamics models. Based on that, we aimed to provide a suitable dynamics model to describe the adsorption/desorption dynamic characteristic of sediments on nitrogen and phosphorus in the north Grand Canal, as well as figure out the influence aspects of the model parameters. The research showed that: 1) Three typical stages, which were quick reaction stage, slow reaction stage and equilibrium stage, were obviously showed in adsorption and desorption curves of nitrogen or phosphorus on sediments in the north Grand Canal; $60 \%$ of the total adsorption and desorption amount occurred in the first stage $(0-0.5 \mathrm{~h})$, and the adsorption rate is higher than the desorption rate. 2) equilibrium adsorption/desorption quantity of every typical sections in the north Grand Canal were difference, the order of equilibrium adsorption quantity is: $S_{\text {(Yulin Village) }}>S_{\text {(Tugou) }}>S_{\text {(Hehe Station) }}$, the order of equilibrium adsorption quantity for ammonia nitrogen is: $S_{\text {(Tugou) }}>S_{\text {(Yulin Village) }}>S_{\text {(Hehe Station) }}$, for phosphate: $S_{\text {(Hehe Station) }}>S_{\text {(Tugou) }}>S_{\text {(Yulin Village) }}$; and chemical adsorption was the main interaction; with the depth increasing, the adsorption rate and desorption rate were decreased, and the upper 0-20 cm has a higher adsorption ability. 3) Lagergren second-order kinetic model performed the best fitting result with the experiment data, and the regression equations are $k_{2}=S_{\max }^{-0.369}+0.163 ; q_{\mathrm{e}}=0.022 S_{\max }+18.077 K_{\mathrm{f}}+41.947$. Through the simulation solution, we obtained that there would be $6 \%-42 \%$ of phosphate and $52 \%-80 \%$ of nitrogen detaching from the sediments by desorption process at $400 \mathrm{mg} / \mathrm{L}$ nitrogen and phosphate concentrations, which will probably cause recontamination to the overlying
\end{abstract}

* 水利部公益性行业科研专项经费项目 (201401054) 资助. 2017-04-19 收稿; 2017-07-02 收修改稿. 胡洁蕴 (1992 ), 女, 硕士研究生; E-mail: hjy992425@cau.edu.cn.

** 通信作者;E-mail: lishuqin@ cau.edu.cn. 
water and pollution risk to ground water through river water percolation.

Keywords: North Grand Canal; sediments; nitrogen; phosphorus; adsorption; desorption ; adsorption rate

北运河是北京市污水排污的主要水系,年排污量占到北京市河流总排污量的 $76 \%^{\left[{ }^{[1]}\right.}$, 已经成为全市地 表水环境质量最差的水系, 其城市排水河道、郊区及城镇下游河道水质多为劣 $\mathrm{V}$ 类. 河道沉积物是水生态系 统的重要组成部分, 是污染物的源和汇 ${ }^{[2]}$, 其对氮、磷的吸附/解吸能力及其动力学过程会对水生态系统健 康产生直接影响. 研究北运河河道沉积物对氮、磷的吸附/解吸特征, 对于揭示北运河河道沉积物对典型污 染物的净化能力以及解决北运河水污染问题具有重要意义.

近年来, 氮、磷等污染物在沉积物中的吸附/解吸特性研究得以大量开展, 但这些研究主要集中在湖泊、 水库等封闭区域, 对于北运河这种城市纳污型河流的研究相对较少 ${ }^{[3-4]}$. 研究表明, 河道沉积物在沉积环境 与沉积时间作用下,有机质含量、颗粒大小、氮磷含量及微生物种类等均会随深度出现时空变化 ${ }^{[5-6]}$, 对氮、 磷的吸附/解吸行为产生重要影响, 进而可能导致不同深度沉积物对氮、磷的吸附/解吸适用的最优动力学 模型不一致. 目前, 常用的污染物在沉积物中的吸附/解吸动力学模型主要包括 Lagergren一级和二级动力 学模型、Weber-Morris 扩散模型和 Elovich 模型 ${ }^{[7-10]}$. 李北罡等 ${ }^{[11]}$ 和李志洪等 ${ }^{[12]}$ 分别研究黄河上游和上海城 市河道沉积物对氮、磷的吸附/解吸动力学发现 Lagergren 二级动力学模型优于一级动力学模型和 WeberMorris 扩散模型; 邵学新等 ${ }^{[13]}$ 和蒋增杰等 ${ }^{[14]}$ 提出 Elovich 模型适用于模拟在初始低浓度磷酸盐溶液中沉积 物对磷的吸附动力学; 此外, 孙大志等 ${ }^{[15-16]}$ 在沈阳张士灌区采用 ExpAssoc 模型模拟氨氮在土壤中的吸附/解 吸过程, 得到较好的模拟效果. 通过对比国内外相关学者的研究结果, 发现不同地域不同类型的沉积物对 氮、磷的吸附/解吸适用的动力学模型不一致. 并且针对于最优动力学模型的研究, 目前仍停留在对比多种 模型模拟的相关系数大小的方法上, 对最优动力模型参数的影响因素研究相对较少. 陈春瑜等 ${ }^{[17]}$ 通过研究 滇池表层沉积物对磷的吸附动力学特征, 提出模型参数的变化规律与沉积物的本底值有关, 但该研究仅止 步于定性说明, 缺少进一步的定量研究, 因此, 研究影响动力学模型参数的因素尤为迫切.

基于此, 胡洁蕴等 ${ }^{[18]}$ 进行了北运河 3 个典型断面沉积物对氨氮的吸附/解吸热力学行为特征研究, 为进 一步探究北运河沉积物对特征污染物的吸附/解吸过程及其动力学特征, 本文继续进行北运河 3 个典型河 道断面 $0 \sim 60 \mathrm{~cm}$ 深度沉积物对氨氮和磷酸盐的吸附/解吸批平衡静态试验研究, 采用多种动力学模型进行 吸附/解吸动力学特性分析, 提出适用于北运河沉积物吸附/解吸特性的动力学模型, 并对模型参数的主要 影响因素进行探讨,为北运河水环境质量改善与河道治理提供理论依据.

\section{1 材料与方法}

\section{1 试验材料}

本试验所用沉积物的采集方式和理化性质及其测试方式与文献 $[18]$ 一致, 沉积物的干密度、质量含水 率、有机质质量分数及氨氮质量分数见文献 $[18]$ 中表 1 , 未涉及的理化性质如表 1 所示, 其中阳离子交换容 量 $(w(\mathrm{CEC}))$ 采用乙酸铵交换法测定; 铑离子的质量分数 $(w(\mathrm{Ca}))$ 采用 TAS-986 型火焰原子吸收分光光度 法测定; 有效磷的质量分数 $(w(\mathrm{P}))$ 采用碳酸氢钠提取 - 钼锑抗比色法测定; 硫酸根离子的质量分数 $\left(w\left(\mathrm{SO}_{4}^{2-}\right)\right)$ 采用 EDTA 间接络合滴定法测定 ${ }^{[19]}$.

\section{2 吸附/解吸批平衡静态法}

1.2.1 批平衡静态法吸附试验 称取 $10 \mathrm{~g}$ 供试沉积物, 加人 $25 \mathrm{ml}$ 浓度为 $400 \mathrm{mg} / \mathrm{L}$ 的氨氮或磷酸盐标准溶 液, 分别振荡 $1 、 5 、 10 、 15$ 和 $20 \mathrm{~min}$ 和 $0.5 、 1.5 、 2 、 3 、 6 、 15$ 和 $24 \mathrm{~h}\left(150\right.$ 转 $\left./ \mathrm{min}, 25^{\circ} \mathrm{C}\right)$. 振荡结束后, 用滤纸过 滤上清液, 得到吸附待测溶液, 采用 AMS-Alliance FUTURA 连续流动分析仪测定溶液中氨氮或磷酸盐的质 量浓度, 利用初始质量浓度与测试值的差值计算单位质量沉积物对氨氮或磷酸盐的吸附质量 ${ }^{[18]}$. 作 $Q-t$ 关 系曲线, 确定吸附平衡时间, 得到吸附动力学曲线.

1.2.2 批平衡静态法解吸试验 称取 $10 \mathrm{~g}$ 供试沉积物, 加人 $25 \mathrm{ml}$ 浓度为 $400 \mathrm{mg} / \mathrm{L}$ 的氨氮或磷酸盐标准溶 液, 进行振荡吸附 $\left(150\right.$ 转 $\left./ \mathrm{min}, 25^{\circ} \mathrm{C}\right)$, 达到吸附平衡时间后, 去除上清液, 并称取过滤过程中损失的沉积物 质量, 加人 $25 \mathrm{ml}$ 去离子水, 在相同的振荡条件下分别振荡 $5 、 10 \mathrm{~min}$ 和 $0.5 、 1 、 2 、 3 、 5 、 10 、 24 、 48 \mathrm{~h}$, 振荡结束 
表 1 各典型断面 $0 \sim 60 \mathrm{~cm}$ 深度沉积物的理化性质

Tab.1 Physical property of 0-60 cm depth's sediments in typical sections

\begin{tabular}{|c|c|c|c|c|c|c|c|c|c|}
\hline \multirow[b]{2}{*}{ 断面 } & \multirow{2}{*}{$\begin{array}{l}\text { 沉积物 } \\
\text { 深度/cm }\end{array}$} & \multirow{2}{*}{$\begin{array}{l}w(\mathrm{CEC}) / \\
(\mathrm{cmol} / \mathrm{kg})\end{array}$} & \multicolumn{4}{|c|}{ 体积分数/\% } & \multirow{2}{*}{$\begin{array}{l}w(\mathrm{Ca}) / \\
(\mathrm{mg} / \mathrm{kg})\end{array}$} & \multirow{2}{*}{$\begin{array}{c}w(\mathrm{P}) / \\
(\mathrm{mg} / \mathrm{kg})\end{array}$} & \multirow{2}{*}{$\begin{array}{r}w\left(\mathrm{SO}_{4}^{2-}\right) \\
(\mathrm{mg} / \mathrm{g})\end{array}$} \\
\hline & & & $\begin{array}{c}<0.002 \\
\mathrm{~mm}\end{array}$ & $\begin{array}{c}0.002 \sim 0.02 \\
\mathrm{~mm}\end{array}$ & $\begin{array}{c}0.02 \sim 0.2 \\
\mathrm{~mm}\end{array}$ & $\begin{array}{c}0.2 \sim 2 \\
\mathrm{~mm}\end{array}$ & & & \\
\hline \multirow[t]{3}{*}{ 土沟 } & $0 \sim 20$ & 8.40 & 3.13 & 19.18 & 46.47 & 31.21 & 106.46 & 9.16 & 0.57 \\
\hline & $20 \sim 40$ & 5.84 & 1.99 & 12.46 & 61.39 & 24.17 & 105.34 & 1.69 & 1.96 \\
\hline & $40 \sim 60$ & 7.83 & 2.54 & 14.05 & 56.57 & 26.84 & 48.88 & 4.07 & 0.68 \\
\hline \multirow[t]{3}{*}{ 榆林庄 } & $0 \sim 20$ & 8.99 & 4.71 & 45.03 & 43.40 & 6.86 & 238.27 & 44.57 & 3.06 \\
\hline & $20 \sim 40$ & 6.63 & 7.86 & 33.94 & 40.34 & 17.87 & 226.89 & 42.64 & 0.64 \\
\hline & $40 \sim 60$ & 5.92 & 5.17 & 25.60 & 54.07 & 15.15 & 73.61 & 2.07 & 0.47 \\
\hline \multirow[t]{3}{*}{ 和合站 } & $0 \sim 20$ & 6.03 & 1.16 & 6.37 & 50.91 & 41.56 & 65.35 & 8.43 & 1.22 \\
\hline & $20 \sim 40$ & 8.45 & 0.36 & 3.98 & 49.18 & 46.49 & 119.18 & 0.46 & 1.29 \\
\hline & $40 \sim 60$ & 5.34 & 0.43 & 3.89 & 46.32 & 49.36 & 350.59 & 8.79 & 2.19 \\
\hline
\end{tabular}

后, 用滤纸过滤上清液, 得到解吸待测溶液, 采用流动分析仪测定溶液中氨氮或磷酸盐的质量浓度, 利用测 试值计算单位质量沉积物对氨氮或磷酸盐的解吸质量 ${ }^{[18]}$. 作 $Q-t$ 关系曲线, 确定解吸平衡时间, 得到解吸 动力学曲线.

本研究中所有处理均设置 3 组平行试验.

\section{3 数据分析}

1.3.1 动力学模型 利用 Lagergren 一级和二级动力学模型 ${ }^{[7]}$ 、Weber-Morris 扩散模型 ${ }^{[8]}$ 、 Elovich 模型 ${ }^{[9-10]}$ 和 ExpAssoc 模型 ${ }^{[15-16]} 5$ 个动力学模型对试验数据进行拟合.

1) Lagergren 一级动力学模型:

$$
\lg \left(q_{\mathrm{e}}-q_{\mathrm{t}}\right)=\lg q_{\mathrm{e}}-\frac{k_{1}}{2.303} \cdot t
$$

式中, $t$ 为吸附或解吸时间 $(\mathrm{h}), q_{\mathrm{t}}$ 为动力吸附或解吸量 $(\mathrm{mg} / \mathrm{kg}), q_{\mathrm{e}}$ 为平衡吸附或解吸量 $(\mathrm{mg} / \mathrm{kg}), k_{1}$ 为一 级吸附或解吸速率常数 $(\mathrm{mg} /(\mathrm{kg} \cdot \mathrm{h}))$.

2) Lagergren 二级动力学模型:

$$
\frac{t}{q_{\mathrm{t}}}=\frac{1}{k_{2} \cdot q_{\mathrm{e}}^{2}}+\frac{1}{q_{\mathrm{e}}} \cdot t
$$

式中, $k_{2}$ 为二级吸附或解吸速率常数 $(\mathrm{mg} /(\mathrm{kg} \cdot \mathrm{h}))$.

3 ) Weber-Morris 扩散模型:

$$
q_{\mathrm{t}}=a_{1}+k_{3} \cdot t^{1 / 2}
$$

式中, $k_{3}$ 为颗粒内扩散速率常数 $\left(\mathrm{mg} /\left(\mathrm{kg} \cdot \mathrm{h}^{1 / 2}\right)\right), a_{1}$ 为吸附或解吸常数.

4) Elovich 模型:

$$
q_{\mathrm{t}}=a_{2}+b_{1} \cdot \ln t
$$

式中, $a_{2}$ 为初始吸附或解吸速率 $(\mathrm{mg} /(\mathrm{kg} \cdot \mathrm{h})) ; b_{1}$ 为吸附或解吸常数, 表示吸附或解吸强度.

5) ExpAssoc 模型:

$$
q_{\mathrm{t}}=A_{1}\left(1-\mathrm{e}^{-t / a_{3}}\right)+A_{2}\left(1-\mathrm{e}^{-t / b_{2}}\right)
$$

式中, $A_{1} 、 A_{2} 、 a_{3}$ 和 $b_{2}$ 均为动力吸附或解吸常数.

1.3.2 相关性分析 使用 SPSS 19.0 软件对试验影响因素进行相关性分析.

\section{2 结果与讨论}

\section{1 沉积物对氨氮和磷酸盐的吸附动力学过程分析}

氨氮与磷酸盐在各断面沉积物中的吸附过程表现为快反应阶段 $(0 \sim 0.5 \mathrm{~h})$ 一慢反应阶段一平衡阶段 
(6 15 h 之后) 3 个阶段 (图 1); 这是由于介质对污染物的吸附过程通常表现为颗粒外部扩散、颗粒内部扩 散及吸附反应 3 个连续阶段; 快反应阶段属于颗粒外部扩散阶段,氮、磷主要附着在沉积物表面, 吸附位点 数量较多, 供大于求, 导致各深度沉积物之间吸附量的差异较小, 且氮、磷在向大孔隙中扩散时受到的阻力 小,吸附较快,基本在 $0 \sim 0.5 \mathrm{~h}$ 之内完成平衡吸附量的 $60 \%$; 慢反应阶段属于颗粒内部扩散阶段,主要是氮、 磷开始从沉积物大孔隙中慢慢向微孔隙中移动, 受到的阻力增加, 并且沉积物的吸附位点被快反应阶段的 大量氮、磷占据而减少, 并伴随着吸附反应, 导致吸附进行较慢, 持续较长, 一般在约 $6 \sim 15 \mathrm{~h}$ 后趋于平衡; 这 种现象与张树楠等 ${ }^{[20]}$ 和方媛媛等 ${ }^{[21]}$ 的研究结论一致. 在 $400 \mathrm{mg} / \mathrm{L}$ 氮、磷浓度下, 随着吸附时间的增加, 各 处理沉积物对氮、磷吸附量的差异逐渐显现出来, 各断面平衡吸附量表现为 $S_{\text {(榆林庄) }}>S_{\text {(土沟) }}>S_{\text {(和合站) }}$, 同一断 面各深度平衡吸附量表现为 $S_{(0 \sim 20 \mathrm{~cm})}>S_{(20 \sim 40 \mathrm{~cm})}>S_{(40 \sim 60 \mathrm{~cm})}$, 表明 $0 \sim 20 \mathrm{~cm}$ 表层沉积物对氨氮和磷酸盐的吸附 能力较大, 这与胡洁蕴等 ${ }^{[18]}$ 研究不同浓度梯度下各深度沉积物对氨氮的吸附量得到的规律一致. 这主要是 由于表层沉积物含有较多的黏粒, 比表面积大, 吸附能力强, 且有机质含量较高, 可为氮、磷的吸附提供较多 的吸附位点, 促进氮、磷在表层沉积物中的吸附 ${ }^{[22]}$.



图 1 氨氮 $(a)$ 和磷酸盐 $(b)$ 在典型断面各深度沉积物中的吸附动力学过程

Fig.1 Adsorption kinetics of ammonia nitrogen (a) and phosphorus (b) at various depth's sediments of typical sections

单位质量吸附剂在单位时间内对吸附质的吸附量称为吸附速率 ${ }^{[17,23]}$. 从表 2 中可以看出, 各断面不同 深度沉积物的吸附速率随时间的增加而降低, 在 $0 \sim 0.5 \mathrm{~h}$ 的吸附速率最大, 对氨氮的吸附速率为 212.75 $599.20 \mathrm{mg} /(\mathrm{kg} \cdot \mathrm{h})$, 对磷酸盐的吸附速率为 $268.80 \sim 1150.55 \mathrm{mg} /(\mathrm{kg} \cdot \mathrm{h})$. 土沟和榆林庄断面沉积物对磷酸 盐的吸附速率是氨氮吸附速率的 2 倍左右, 而和合站二者却相差不大, 这主要是因为沉积物对磷酸盐的吸 附速率与小于 $0.063 \mathrm{~mm}$ 细颗粒的体积百分比含量呈现较好的正相关关系 ${ }^{[24]}$. 小于 $0.063 \mathrm{~mm}$ 的细颗粒含量 在土沟和榆林庄断面沉积物中占 70\% 80\%, 而在和合站断面仅占 50\%, 由此可知, 和合站断面颗粒较大, 导 致其沉积物对磷的吸附速率相对降低, 而对氨氮的吸附速率影响不明显. 沉积物吸附氨氮在 $6 \mathrm{~h}$ 后的速率和 吸附磷酸盐在 $15 \mathrm{~h}$ 后的速率均小于 $0 \sim 0.5 \mathrm{~h}$ 速率的 $2 \%$, 表明在 6 和 $15 \mathrm{~h}$ 之后, 沉积物对氨氮和磷酸盐的吸 附速率较小, 可初步认为沉积物对氨氮和磷酸盐的吸附已经达到动态平衡状态, 这一结论与蒋增杰等 ${ }^{[14]}$ 和 季现超等 ${ }^{[25]}$ 的研究结论相吻合, 但沉积物对磷酸盐平衡吸附的时间要早于李北罡等 ${ }^{[11]}$ 的研究结论, 其原因 可能是黄河水体含沙量大, 沉积物颗粒较粗, 吸附速率慢, 达到平衡状态耗时长.

氨氮和磷酸盐在各深度断面沉积物上吸附过程的模型拟合结果见附表 1 、附表 2. 各断面沉积物对氨氮的 吸附拟合效果表现为: Lagergren 二级动力学模型 $>$ ExpAssoc 模型 $>$ Elovich 模型 $>$ Lagergren 一级动力学模型 $>$ Weber-Morris 扩散模型; 对磷酸盐的吸附拟合效果表现为: Lagergren 二级动力学模型>Lagergren一级动力学 模型> ExpAssoc 模型>Weber-Morris 扩散方程 > Elovich 模型. 相较而言, Lagergren 二级动力学模型拟合最 
优, 相关系数在 0.99 以上, 通过该模型推算出在 $400 \mathrm{mg} / \mathrm{L}$ 氮、磷浓度下, 氨氮平衡吸附量为 $150.00 \sim 357.70$ $\mathrm{mg} / \mathrm{kg}$, 磷酸盐的平衡吸附量为 $277.78 \sim 909.09 \mathrm{mg} / \mathrm{kg}$, 该结论与图 1 所示的平衡吸附量较为接近 ${ }^{[12,21]}$. 研究 表明, 沉积物吸附磷酸盐主要是因为沉积物颗粒表面的吸附点位周围聚集了大量的无机磷酸根离子, 可与 沉积物颗粒表面吸附点位上的羟基 $(\mathrm{M}-\mathrm{OH})$ 或水合基 $\left(\mathrm{M}-\mathrm{OH}_{2}\right)$ 迅速地进行配位体交换 ${ }^{[26]}$; 吸附氨氮主要是 因为沉积物表面吸附点位的离子和 $\mathrm{NH}_{4}^{+}$之间产生静电力以及沉积物内部阳离子与 $\mathrm{NH}_{4}^{+}$进行离子交换 ${ }^{[27]}$. Lagergren 二级动力学模型较为准确地描述了氮、磷污染物在各深度沉积物中的化学吸附过程 ${ }^{228]}$. 但是, 王 丽香等 ${ }^{[29]}$ 研究东部河网河道沉积物对磷的吸附时, 发现 Elovich 模型的拟合结果优于 Lagergren二级动力学 模型, 这主要是因为王丽香等使用了简化的 Lagergren 二级动力学模型, 其表达式为: $1 / c=1 / c_{0}+k \cdot t$, 等式 左边忽略了时间 $t$, 导致该模型拟合结果变差; 而 Elovich 模型的拟合结果优于本研究, 是因为王丽香等的试 验初始磷酸盐溶液浓度只有 $1.0 \mathrm{mg} / \mathrm{L}$, 但本研究初始磷酸盐溶液浓度为 $400 \mathrm{mg} / \mathrm{L}$, 在高初始浓度下沉积物 对磷的吸附过程中可能产生磷酸铝盐沉淀, 不易于磷在沉积物颗粒内部扩散, 导致本研究中 Elovich 模型模 拟效果差. 总体而言, Lagergren二级动力学模型拟合效果最优, 在 $400 \mathrm{mg} / \mathrm{L}$ 氮、磷浓度下, 对氨氮的吸附速 率常数为 $0.0050 \sim 0.1718 \mathrm{mg} /(\mathrm{kg} \cdot \mathrm{h})$, 对磷酸盐的吸附速率常数为 $0.0008 \sim 0.0068 \mathrm{mg} /(\mathrm{kg} \cdot \mathrm{h})$, 这与其他研 究者得到的动力吸附速率常数范围相一致 ${ }^{[1,15,20]}$.

表 2 各典型断面 $0 \sim 60 \mathrm{~cm}$ 深度沉积物对氨氮和磷酸盐不同时间段的吸附速率 $(\mathrm{mg} /(\mathrm{kg} \cdot \mathrm{h}))$

Tab.2 Adsorption velocity of 0-60 cm depth's sediments for ammonia nitrogen and phosphorus during different times in selected sections

\begin{tabular}{|c|c|c|c|c|c|c|c|c|c|c|c|c|c|}
\hline \multirow{3}{*}{ 断面 } & \multirow{3}{*}{$\begin{array}{c}\text { 沉积物 } \\
\text { 深度/cm }\end{array}$} & \multicolumn{12}{|c|}{ 采样时间段/h } \\
\hline & & \multicolumn{2}{|c|}{$0 \sim 0.5$} & \multicolumn{2}{|c|}{$0.5 \sim 1$} & \multicolumn{2}{|c|}{$1 \sim 3$} & \multicolumn{2}{|c|}{$3 \sim 6$} & \multicolumn{2}{|c|}{$6 \sim 15$} & \multicolumn{2}{|c|}{$15 \sim 24$} \\
\hline & & 氨氮 & 磷酸盐 & 氨氮 & 磷酸盐 & 氨氮 & 磷酸盐 & 氨氮 & 磷酸盐 & 氨氮 & 磷酸盐 & 氨氮 & 磷酸盐 \\
\hline \multirow[t]{3}{*}{ 土沟 } & $0 \sim 20$ & 269.95 & 462.40 & 89.05 & 164.45 & 26.54 & 109.73 & 12.02 & 28.48 & 0.64 & 18.25 & 0.16 & 9.64 \\
\hline & $20 \sim 40$ & 262.35 & 496.00 & 30.15 & 67.20 & 1.90 & 31.36 & 8.06 & 26.89 & 4.46 & 13.10 & 0.18 & 3.96 \\
\hline & $40 \sim 60$ & 212.75 & 318.50 & 17.25 & 51.65 & 10.20 & 32.78 & 8.28 & 17.57 & 3.88 & 15.71 & 2.13 & 2.99 \\
\hline \multirow[t]{3}{*}{ 榆林庄 } & $0 \sim 20$ & 599.20 & 1150.55 & 27.10 & 85.00 & 16.45 & 53.33 & 9.88 & 69.64 & 0.28 & 5.70 & 0.19 & 2.62 \\
\hline & $20 \sim 40$ & 344.40 & 649.90 & 11.45 & 116.65 & 15.45 & 35.93 & 5.25 & 12.38 & 2.50 & 11.78 & 0.63 & 5.22 \\
\hline & $40 \sim 60$ & 298.25 & 494.45 & 9.25 & 115.50 & 9.66 & 34.00 & 29.48 & 87.06 & 1.77 & 15.70 & 0.99 & 5.02 \\
\hline \multirow[t]{3}{*}{ 和合站 } & $0 \sim 20$ & 344.45 & 302.55 & 13.10 & 36.25 & 8.00 & 9.68 & 8.78 & 12.68 & 1.89 & 13.36 & 0.43 & 2.87 \\
\hline & $20 \sim 40$ & 287.80 & 268.80 & 4.74 & 35.65 & 5.56 & 33.40 & 0.73 & 15.16 & 0.31 & 1.65 & 0.55 & 0.69 \\
\hline & $40 \sim 60$ & 240.66 & 291.55 & 26.46 & 19.18 & 3.45 & 15.41 & 1.96 & 7.45 & 0.13 & 4.07 & 0.31 & 0.08 \\
\hline
\end{tabular}

\section{2 沉积物对氨氮和磷酸盐的解吸动力学过程分析}

沉积物对氮、磷的动力解吸过程与吸附过程相似, 存在快反应阶段 $(0 \sim 0.5 \mathrm{~h})$ 一慢反应阶段一平衡阶段 (6 10 h 之后) 3 个反应阶段 (图 2). 各断面的解吸量随深度增加而逐渐减小, $0 \sim 20 \mathrm{~cm}$ 表层沉积物的解吸 量最大, 与胡洁蕴等 ${ }^{[18]}$ 在不同浓度梯度下表层沉积物对氨氮的解吸量最大的研究结论一致; 各断面沉积物


相较于氨氮, 和合站断面沉积物对磷酸盐的平衡解吸量较大, 主要是因为和合站断面沉积物的砂粒含量较 高, 砂粒中腐殖质含量远远低于黏粒和粉粒, 不易与磷形成团聚体结构, 导致沉积物对磷的固持能力削弱, 造成和合站断面沉积物对磷酸盐的平衡解吸量较大 ${ }^{[30]}$.

各典型断面 0 60 cm 深度沉积物对氨氮、磷酸盐的解吸速率 (表 3) 同吸附速率 (表 2) 规律一致, 表现 为随时间的增加解吸速率降低, 在 $0 \sim 0.5 \mathrm{~h}$ 解吸速率最大, 氨氮解吸速率为 $152.33 \sim 261.16 \mathrm{mg} /(\mathrm{kg} \cdot \mathrm{h})$, 磷酸 盐解吸速率为 $39.22 \sim 181.75 \mathrm{mg} /(\mathrm{kg} \cdot \mathrm{h})$; 对氮、磷的解吸速率均小于吸附速率, 且解吸平衡总量低于吸附平 衡总量, 解吸过程滞后于吸附过程 ${ }^{[00-31]}$. 对比氨氮与磷酸盐的解吸速率 (表 3 ), 发现沉积物对磷酸盐的解吸 速率低于氨氮, 可能是因为磷与沉积物在吸附过程中形成了难以转化的吸附态磷、铁结合态磷、铝结合态磷 及有机磷等 ${ }^{[32-33]}$, 导致解吸速率降低, 这表明该区域沉积物因自然释磷而引起的水体磷富营养化的风险性 


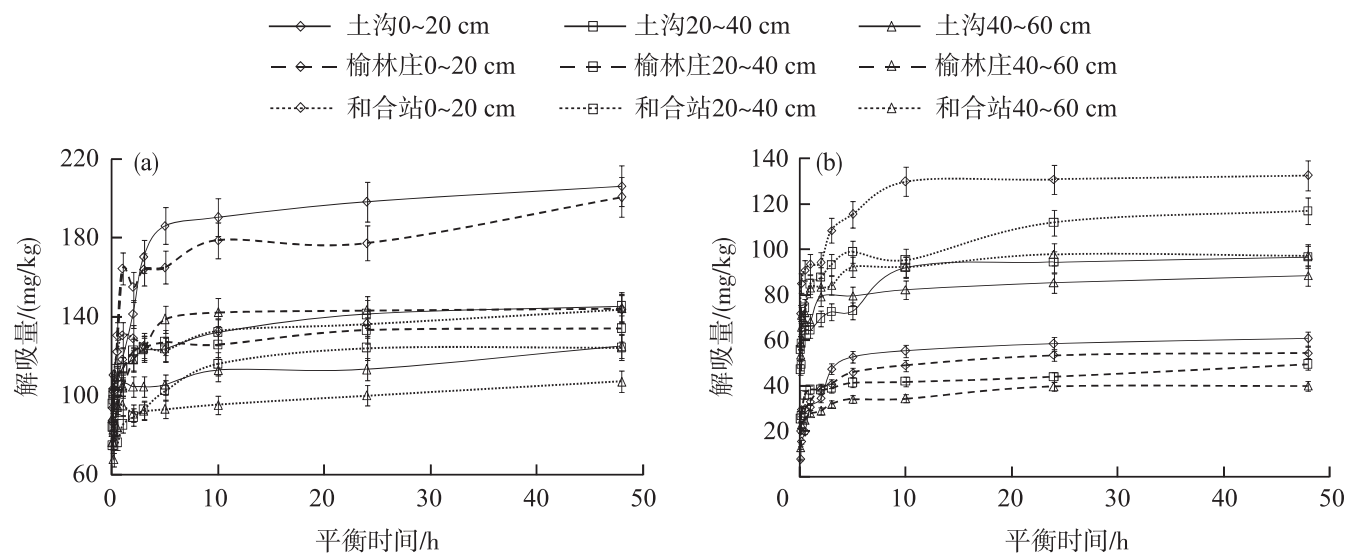

图 2 氨氮 (a) 和磷酸盐 (b) 在典型断面各深度沉积物中的解吸动力学过程

Fig. 2 Desorption kinetics of ammonia nitrogen (a) and phosphorus (b) at various depth's sediments of typical sections

较小. $6 \mathrm{~h}$ 后沉积物的解吸速率小于 $1.00 \mathrm{mg} /(\mathrm{kg} \cdot \mathrm{h})$, 沉积物对氨氮和磷酸盐的解吸趋近于平衡状态, 该平 衡时间与陈建平等 ${ }^{[34]}$ 和徐明德等 ${ }^{[35]}$ 研究得到的水稻田土和长江沉积物对氨氮、磷酸盐的解吸平衡时间较 为一致.

氨氮和磷酸盐在各断面沉积物上解吸过程的模型拟合结果见附表 3 和附表 4. 各断面沉积物对氨氮动力 解吸拟合效果表现为: Lagergren 二级动力学模型 $>$ ExpAssoc 模型 $>$ Elovich 模型 $>$ Lagergren 一级动力学模型 $>$ Weber-Morris 扩散模型; 对磷酸盐的解吸拟合效果表现为: Lagergren二级动力学模型 > Elovich 模型 > ExpAssoc 模型>Lagergren 一级动力学模型 $>$ Weber-Morris 扩散模型. Lagergren 二级动力学模型对各深度沉积 物氮、磷解吸过程的拟合最优 $\left(R^{2}>0.99\right)$, 通过 Lagergren 二级动力学拟合, 得到在 $400 \mathrm{mg} / \mathrm{L}$ 氮、磷浓度下, 氨 氮平衡解吸量为 $106.38 \sim 208.33 \mathrm{mg} / \mathrm{kg}$, 磷酸盐平衡解吸量为 $40.32 \sim 133.33 \mathrm{mg} / \mathrm{kg}$, 模型推算得到的平衡解 吸量与图 2 所示的平衡解吸量结果较为接近; 对氨氮的解吸速率常数为 $0.0081 \sim 0.0342 \mathrm{mg} /(\mathrm{kg} \cdot \mathrm{h})$, 对磷酸 盐的解吸速率常数为 $0.012 \sim 0.041 \mathrm{mg} /(\mathrm{kg} \cdot \mathrm{h})$.

表 3 各典型断面 $0 \sim 60 \mathrm{~cm}$ 深度沉积物对氨氮和磷酸盐不同时间段的解吸速率 $(\mathrm{mg} /(\mathrm{kg} \cdot \mathrm{h}))$

Tab.3 Desorption velocity of 0-60 cm depth's sediments for ammonia nitrogen and phosphorus during different times in selected sections

\begin{tabular}{|c|c|c|c|c|c|c|c|c|c|c|c|c|c|}
\hline \multirow{3}{*}{ 断面 } & \multirow{3}{*}{$\begin{array}{c}\text { 沉积物 } \\
\text { 深度/cm }\end{array}$} & \multicolumn{12}{|c|}{ 采样时间段/h } \\
\hline & & \multicolumn{2}{|c|}{$0 \sim 0.5$} & \multicolumn{2}{|c|}{$0.5 \sim 1$} & \multicolumn{2}{|c|}{$1 \sim 3$} & \multicolumn{2}{|c|}{$3 \sim 6$} & \multicolumn{2}{|c|}{$6 \sim 15$} & \multicolumn{2}{|c|}{$15 \sim 24$} \\
\hline & & 氨氮 & 磷酸盐 & 氨氮 & 磷酸盐 & 氨氮 & 磷酸盐 & 氨氮 & 磷酸盐 & 氨氮 & 磷酸盐 & 氨氮 & 磷酸盐 \\
\hline \multirow[t]{3}{*}{ 土沟 } & $0 \sim 20$ & 217.20 & 39.22 & 19.59 & 22.19 & 26.11 & 8.40 & 2.81 & 1.13 & 0.58 & 0.22 & 0.33 & 0.10 \\
\hline & $20 \sim 40$ & 210.65 & 129.00 & 16.84 & 1.02 & 4.76 & 4.06 & 1.29 & 2.79 & 0.65 & 0.17 & 0.16 & 0.09 \\
\hline & $40 \sim 60$ & 168.45 & 136.24 & 42.10 & 9.28 & 1.19 & 2.86 & 0.31 & 0.56 & 0.02 & 0.21 & 0.49 & 0.14 \\
\hline \multirow[t]{3}{*}{ 榆林庄 } & $0 \sim 20$ & 261.16 & 59.64 & 67.80 & 6.70 & 2.09 & 3.85 & 0.20 & 1.16 & 0.10 & 0.32 & 0.97 & 0.04 \\
\hline & $20 \sim 40$ & 219.39 & 72.04 & 8.51 & 4.52 & 5.17 & 0.36 & 0.23 & 0.41 & 0.52 & 0.15 & 0.05 & 0.23 \\
\hline & $40 \sim 60$ & 189.15 & 49.96 & 47.88 & 5.67 & 10.04 & 2.04 & 2.59 & 0.37 & 0.07 & 0.38 & 0.04 & 0.01 \\
\hline \multirow[t]{3}{*}{ 和合站 } & $0 \sim 20$ & 245.10 & 181.75 & 16.25 & 5.16 & 2.62 & 7.48 & 1.05 & 3.08 & 0.25 & 0.06 & 0.31 & 0.07 \\
\hline & $20 \sim 40$ & 152.33 & 148.85 & 18.46 & 21.08 & 3.85 & 4.19 & 3.29 & 1.18 & 0.58 & 0.30 & 0.01 & 0.21 \\
\hline & $40 \sim 60$ & 190.88 & 144.33 & 1.41 & 21.95 & 0.53 & 1.17 & 0.43 & 0.50 & 0.34 & 0.40 & 0.29 & 0.02 \\
\hline
\end{tabular}

解吸比是解吸平衡量与吸附平衡量之比, 用来表征潜在污染风险. 在 $400 \mathrm{mg} / \mathrm{L}$ 氮、磷浓度下, 3 个断面 
沉积物对氨氮的解吸比为 $52 \% \sim 80 \%$; 对磷酸盐的解吸比为 $6 \% \sim 42 \%$, 这表明约有 $52 \% \sim 80 \%$ 的氨氮与 6\% $42 \%$ 的磷酸盐释放引起上覆水体二次污染, 还有可能氮、磷随河水人渗进人下层渗滤介质, 影响地下水环境, 对地下水质具有潜在污染风险 ${ }^{[15,36]}$.

\section{3 氨氮和磷酸盐在沉积物中吸附/解吸的影响因素分析}

典型污染物在沉积物中的吸附/解吸性能会受到沉积物理化性质的影响, 如沉积物干密度 $(d)$ 、质量含 水率 $(\omega)$ 及氮、磷本底值等, 研究通过对沉积物的 $d 、 \omega$ 、黏粒体积分数 $(w$ (Clay))、有机质质量分数 $(w(\mathrm{SOM})) 、 w(\mathrm{CEC}) 、 w(\mathrm{Ca}) 、 w(\mathrm{P})$ 、氨氮的质量分数 $\left(w\left(\mathrm{NH}_{4}^{+}\right)\right)$及 $w\left(\mathrm{SO}_{4}^{2-}\right)$ 共 9 个影响因素与最优模拟模 型—Lagergren二级动力模型参数进行相关分析, 分析各因素对氮、磷动力吸附/解吸性能的影响. 结果表 明,各影响因素与 Lagergren 二级动力模型参数的相关关系并不显著 $($ 表 4).

表 4 沉积物理化性质及热力学模型参数与动力学模型参数的相关性分析

Tab.4 Correlation analysis between sediment physicochemical properties and thermodynamic parameters and dynamic model parameters

\begin{tabular}{cccccccccccccc}
\hline 参数 & $d$ & $\omega$ & $w(\mathrm{SOM})$ & $w(\mathrm{Clay})$ & $w\left(\mathrm{C}_{\mathrm{a}}\right)$ & $w(\mathrm{CEC})$ & $w\left(\mathrm{SO}_{4}^{2-}\right)$ & $w\left(\mathrm{NH}_{4}^{+}\right)$ & $w(\mathrm{P})$ & $n$ & $K_{\mathrm{f}}$ & $S_{\max }$ \\
\hline$k_{2}$ & 0.06 & -0.23 & -0.18 & 0.12 & 0.13 & 0.05 & 0.04 & -0.05 & -0.07 & 0.14 & -0.12 & $-0.34^{*}$ \\
$q_{\mathrm{e}}$ & -0.22 & $0.28^{*}$ & 0.18 & -0.09 & -0.04 & 0.15 & 0.02 & 0.10 & 0.16 & -0.09 & $0.34^{*}$ & $0.73^{* *}$ \\
\hline
\end{tabular}

*表示在 0.05 水平 (双侧) 上显著相关; $* *$ 表示在 0.01 水平 (单侧) 上显著相关.

胡洁蕴等 ${ }^{[18]}$ 研究了氨氮在沉积物中的吸附/解吸热力学行为, 并采用 Frendlich 和 Langmuir 模型对试验 数据进行拟合得到 Frendlich 模型热力学参数 $n$ 和 $K_{\mathrm{f}}$ 以及 Langmuir 模型热力学参数 $S_{\text {max }}$ (参数详见文献 $[18]$ 中的表 2 和表 4). 本文在此基础上, 将氮、磷在沉积物中的吸附/解吸热力学模型参数与动力学模型参数进 行相关分析, 并探讨了最优模拟模型—Lagergren 二级动力模型参数与 Frendlich 模型、Langmuir 模型参数 之间的关系, 沉积物对磷酸盐的吸附/解吸热力学模型参数见表 5 . 研究发现, Lagergren二级动力模型的 $k_{2}$ 值与 Langmuir 模型模拟的吸附/解吸理论最大值 $S_{\text {max }}(-0.34, P<0.05)$ 呈现显著中等负相关, $q_{\mathrm{e}}$ 与 Frendlich 模型模拟的吸附/解吸常数 $K_{\mathrm{f}}(0.34, P<0.05)$ 、 Langmuir 模型模拟的吸附/解吸理论最大值 $S_{\text {max }}(0.73, P<$ $0.01)$ 呈显著中等正相关. 与针对 $k_{2} 、 q_{\mathrm{e}}$ 与 $K_{\mathrm{f}} 、 S_{\text {max }}$ 呈显著中等相关的性质, 利用 SPSS 19.0 软件进行回归分析, 得到动力学和热力学模型参数之间的关系式: $k_{2}=S_{\text {max }}^{-0.369}+0.163\left(R^{2}=0.334\right) ; q_{\mathrm{e}}=0.022 S_{\text {max }}+18.077 K_{\mathrm{f}}+$ 41.947 $\left(R^{2}=0.678\right) . K_{\mathrm{f}}$ 和 $S_{\text {max }}$ 越大, 表明吸附或解吸能力越强 ${ }^{[18]}, q_{\mathrm{e}}$ 值越大, 对应的数学表达式也具有一样 的规律, 故回归分析所得的关系式建立了热力学和动力学模型参数之间的相互转化关系. 今后学者在研究 北运河沉积物对氨氮、磷酸盐吸附/解吸特征时, 可以根据以上关系式将热力学研究得到的模型参数用于动 力学模型参数值的估算, 省去动力学研究的繁琐试验, 大大减少了研究工作量. 同时, 也为今后学者研究沉 积物对氨氮和磷酸盐吸附/解吸的热力学和动力学之间的关系提供参考.

有研究表明沉积物中 $w(\mathrm{SOM})$ 与 $q_{\mathrm{e}}$ 存在显著正相关关系, 但本研究并未得到显著关系, 主要是因为本 研究所取沉积物的 $w(\mathrm{SOM})$ 低, 并未达到固相物质总质量的 5\%, 导致相关性不显著 ${ }^{[25]}$. 也有研究表明沉积 物中氮、磷本底值越高, 其吸附水体中氮、磷的量和速率就会相应减小, 但是通过相关性分析, 氮、磷本底值 并未对吸附速率和平衡吸附量产生影响, 原因可能是沉积物中氮、磷本底值小于平衡吸附量的 $10 \%$, 还有较 大的吸附空间, 对吸附速率和吸附量限制较小 ${ }^{[24]}$.

目前河道沉积物对氮、磷吸附/解吸的研究中,一方面试验材料主要集中在 $0 \sim 10 \mathrm{~cm}$ 的表层沉积物, 而 在以土壤作为试验材料的研究中深度可达到 $60 \sim 80 \mathrm{~cm}$, 考虑土壤研究中得到吸附/解吸氮、磷随深度变化的 规律, 本研究和胡洁蕴等 ${ }^{[18]}$ 的研究拓展了河道沉积物的研究深度, 探索了 $0 \sim 60 \mathrm{~cm}$ 深度范围内沉积物对氨 氮、磷酸盐的吸附/解吸特征, 并得出了变化规律. 另一方面研究方法多采用批平衡静态法, 而溶质在沉积物 中运移时常常与其他溶质、固相物质及微生物发生相互作用, 如吸附、解吸、交换、生化反应等过程 ${ }^{\left[{ }^{[3]}\right.}$, 显然 用单一的批平衡法得到的试验值与实际情况比较, 结果会相差较远. 故在今后研究中可以借鉴土壤的吸附/ 解吸研究方法, 采用批平衡静态法与动态土柱法相结合来研究河道沉积物对特征污染物的吸附/解吸特征. 
表 5 各典型断面 $0 \sim 60 \mathrm{~cm}$ 深度沉积物对磷酸盐吸附/解吸的热力学模型参数

Tab.5 Thermodynamic model's simulation parameter to adsorption/desorption

behavior of 0-60 cm depth's sediments for phosphorus in selected sections

\begin{tabular}{|c|c|c|c|c|c|c|c|c|c|c|}
\hline \multirow{3}{*}{ 过程 } & \multirow{3}{*}{ 参数 } & \multicolumn{9}{|c|}{ 断面 } \\
\hline & & \multicolumn{3}{|c|}{ 土沟 } & \multicolumn{3}{|c|}{ 榆林庄 } & \multicolumn{3}{|c|}{ 和合站 } \\
\hline & & $0 \sim 20 \mathrm{~cm}$ & $20 \sim 40 \mathrm{~cm}$ & $40 \sim 60 \mathrm{~cm}$ & $0 \sim 20 \mathrm{~cm}$ & $20 \sim 40 \mathrm{~cm}$ & $40 \sim 60 \mathrm{~cm}$ & $0 \sim 20 \mathrm{~cm}$ & $20 \sim 40 \mathrm{~cm}$ & $40 \sim 60 \mathrm{~cm}$ \\
\hline \multirow[t]{3}{*}{ 吸附 } & $n$ & 1.21 & 1.20 & 1.02 & 0.78 & 1.59 & 1.69 & 0.54 & 0.91 & 0.84 \\
\hline & $S_{\max }$ & 15315 & 12722 & 13554 & 27827 & 12553 & 17573 & 10597 & 17459 & 14022 \\
\hline & $K_{\mathrm{f}}$ & 8.61 & 4.05 & 1.36 & 1.11 & 14.25 & 20.45 & 7.71 & 0.48 & 0.27 \\
\hline \multirow[t]{3}{*}{ 解吸 } & $n$ & 0.61 & 0.85 & 0.89 & 1.61 & 0.90 & 0.88 & 1.64 & 0.85 & 0.89 \\
\hline & $S_{\max }$ & 8356.59 & 1623.07 & 1696.90 & 3487.07 & 2236.50 & 1738.54 & 868.85 & 2942.50 & 2608.46 \\
\hline & $K_{\mathrm{f}}$ & 0.003 & 0.11 & 0.11 & 1.50 & 0.17 & 0.13 & 1.70 & 0.12 & 0.15 \\
\hline
\end{tabular}

\section{3 结论}

1) 北运河 3 个典型断面各深度沉积物对氨氮和磷酸盐的吸附/解吸过程是一个复合动力学过程, 总体 可分为 3 个阶段: 快反应阶段一慢反应阶段一平衡阶段; 吸附和解吸主要发生在 $0 \sim 0.5 \mathrm{~h}$ 的快反应阶段, 可完 成吸附或解吸总量的 $60 \%$; 在快反应阶段对氨氮的吸附速率为 $212.75 \sim 599.20 \mathrm{mg} /(\mathrm{kg} \cdot \mathrm{h})$, 对磷酸盐的吸附 速率为 $268.80 \sim 1150.55 \mathrm{mg} /(\mathrm{kg} \cdot \mathrm{h})$, 吸附速率大于解吸速率.

2) 北运河 3 个典型断面各深度沉积物对氮、磷的吸附/解吸性能差异显著, 对氨氮与磷酸盐的平衡吸附

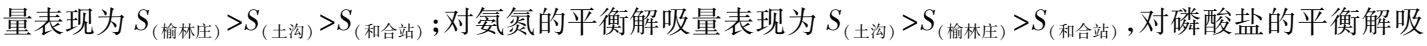
量表现为 $S_{\text {(和合站) }}>S_{\text {(土沟) }}>S_{\text {(榆林庄) }}$, 沉积物对氨氮、磷酸盐的吸附以化学吸附为主, 且平衡吸附与解吸量随断 面深度的增加而减小, $0 \sim 20 \mathrm{~cm}$ 表层沉积物对氮、磷的吸附能力较强.

3) Lagergren 二级动力学模型对各深度沉积物的吸附/解吸过程拟合最优 $\left(R^{2}>0.99\right)$, 模型参数 $k_{2} 、 q_{\mathrm{e}}$ 与 热力学模型参数 $K_{\mathrm{f}} 、 S_{\text {max }}$ 呈现显著中等相关关系, 回归方程为 $k_{2}=S_{\text {max }}^{-0.369}+0.163 ; q_{\mathrm{e}}=0.022 S_{\max }+18.077 K_{\mathrm{f}}+$ 41.947. 该模型模拟得出在 $400 \mathrm{mg} / \mathrm{L}$ 氮、磷浓度下吸附于沉积物中的氮、磷污染物会随着解吸过程平均释放 $52 \% \sim 80 \%$ 的氨氮和 $6 \% \sim 42 \%$ 的磷酸盐, 可能不仅引起上覆水体二次污染, 还随河水下渗对地下水质形成潜 在污染风险.

\section{4 附录}

附表 1 4 见电子版( DOI: 10.18307/2018.0307).

\section{5 参考文献}

[ 1 ] Jing HW, Zhang ZG, Guo J. Water pollution characteristics and pollution sources of Bei Canal river system in Beijing. China Environmental Science, 2013, 33(2) : 319-327. [荆红卫, 张志刚, 郭婧. 北京北运河水系水质污染特征及污染 来源分析. 中国环境科学, 2013, 33(2):319-327.]

[ 2 ] He Y, Chen YX, Zhang YF et al. Role of aerated turbulence in the fate of endogenous nitrogen from malodorous river sediments. Environmental Engineering Science, 2013, 30(1): 11-16.

[ 3 ] Zhang ZB, Wei LL, Gao BY et al. Nutrient release characteristics from the sediments in Nansi Lake. Environmental Chemistry, 2013, 31(2) : 221-225. [ 张志斌, 魏垒垒, 高宝玉等. 南四湖沉积物营养盐释放特性. 环境化学, 2012, 31 (2) : 221-225.]

[ 4 ] Jin G, Onodera S, Amano A et al. Effects of dam construction on sediment phosphorus variation in a semi-enclosed bay of the Seto Inland Sea, Japan. Estuarine Coastal and Shelf Science, 2013, 135(20) : 191-200.

[ 5 ] Wang SJ, Yan YX, Yan M et al. Spatial variations of gravel sediment granularities and their causes in Ganxi Stream of Zhangjiajie. Progress in Geography, 2014, 33(1) : 34-41. [王随继, 间云霞, 颜明等. 张家界甘溪砾石沉积物粒度的 
空间变化及其原因. 地理科学进展, 2014, 33(1) : 34-41.]

[6] Gao WH, Gao S, Wang DD et al. Sediment source information of different catchments in the sedimentary records of the abandoned Yellow River: heavy mineral and geochemical analyses. Scientia Geographica Sinica, 2015, 35(12): 16311639. [高文华, 高抒, 王丹丹等. 废黄河沉积记录中来自不同河流物质的信息——基于重矿物与地球化学元素分 析. 地理科学, 2015, 35(12): 1631-1639.]

[ 7 ] Otero M, Rozada F, Calvo LF et al. Kinetic and equilibrium modelling of the methylene blue removal from solution by adsorbent materials produced from sewage sludges. Biochemical Engineering Journal, 2003, 15(1) : 59-68.

[ 8 ] Bulut Y, Aydin H. A kinetics and thermodynamics study of methylene blue adsorption on wheat shells. Desalination, 2006, 194(1): 259-267.

[ 9 ] Padmesh TVN, Vijayaraghavan K, Sekaran G et al. Batch and column studies on biosorption of acid dyes on fresh water macroalga Azolla filiculoides. Journal of Hazardous Materials, 2005, 125(1/2/3) : 121-129.

[10] Wang Y, Shen ZY, Niu JF et al. Adsorption of phosphorus on sediments from the Three-Gorges Reservoir (China) and the relation with sediment compositions. Journal of Hazardous Materials, 2009, 162(1) : 92-98.

[11] Li BG, Liu PY, Ma Q. Adsorption kinetics of phosphate onto sediments from the upper reaches of the Yellow River. Ecology and Environmental Sciences, 2010, 19(11): 2693-2697. [李北罡, 刘培怡, 马钦. 黄河上游沉积物对磷酸盐的吸 附动力学研究. 生态环境学报, 2010, 19(11): 2693-2697.]

[12] Li ZH, Shen SY, He Y et al. The adsorption and desorption of ammonia-nitrogen in sediment of urban malodorous river. Shanghai Chemical Industry, 2014, 39(5): 1-3. [李志洪, 沈叔云, 何岩等. 城市黑臭河道底泥氨氮吸附解吸性能 研究. 上海化工, 2014, 39(5): 1-3.]

[13] Shao XX, Liang W, Wang M et al. Adsorption characteristics of phosphate on sediment under typical vegetation types in Hangzhou Bay. Soils, 2014, 46(6) : 1032-1038. [邵学新, 梁威, 王蒙等. 杭州湾典型潮滩湿地植物带沉积物磷吸 附特征. 土壤, 2014, 46(6): 1032-1038.]

[14] Jiang ZJ, Wang GH, Fang JG et al. Phosphate adsorption characteristics onto surface sediments from aquaculture area in Sungo Bay. Environmental Science, 2008, 29(12): 3405-3409. [蒋增杰, 王光花, 方建光等. 桑沟湾养殖水域表层沉 积物对磷酸盐的吸附特征. 环境科学, 2008, 29(12): 3405-3409.]

[15] Sun DZ, Li XQ, Pan XF. Ammonia adsorption/desorption behavior in soil. Environmental Science and Technology, 2007, 30(8) : 16-18, 111. [孙大志, 李绪谦, 潘晓峰. 氨氮在土壤中的吸附/解吸动力学行为的研究. 环境科学与技术, $2007, \mathbf{3 0}(8): 16-18,111$.

[16] Sun DZ. Ammonia adsorption/desorption behavior and thermodynamics in soil. Journal of Beihua University: Natural Science, 2007, 8(6) : 493-496. [孙大志. 氨氮在土壤中吸附/解吸的动力学与热力学研究. 北华大学学报: 自然科学 版, 2007, 8(6): 493-496.]

[17] Chen CY, Xu XM, Deng WM et al. Characteristics of phosphorus adsorption on surface sediments of Dianchi Lake. Acta Scientiae Circumstantiae, 2014, 34(12):3065-3075. [陈春瑜, 徐晓梅, 邓伟明等. 滇池表层沉积物对磷的吸附特 征. 环境科学学报, 2014, 34(12): 3065-3075.]

[18] Hu JY, Li SQ, Li YK et al. Study on the adsorption/desorption characteristics of different depth sediments for ammonia nitrogen in urban river. Journal of China Agricultural University, 2017, 22(7): 78-89. [胡洁蕴, 李淑芹, 李云开等. 城 市河道不同深度沉积物对氨氮的吸附-解吸特征. 中国农业大学学报, 2017, 22(7): 78-89.]

[19] The National Agro-Tech Extension and Service Center ed. Soil analysis technical specification: version 2. Beijing: Agricultural Publishing House, 2014: 134-158. [全国农业技术推广服务中心. 土壤分析技术规范: 第 2 版. 北京: 农业出 版社, 2014: 134-158.]

[20] Zhang SN, Jia ZY, Xiao RL et al. Study on phosphorus adsorption characteristic of sediments in an ecological ditch. Environmental Science, 2013, 34(3) : 1101-1106. [张树楠, 贾兆月, 肖润林等. 生态沟渠底泥属性与磷吸附特性研究. 环境科学, 2013, 34(3): 1101-1106.]

[21] Fang YY, Liu LH, Wu LX et al. Study on the adsorption characteristics of nitrogen and phosphorous on five substrates. Applied Chemical Industry, 2016, 45(9): 1619-1623. [方媛瑗, 刘玲花, 吴雷祥等. 5 种填料对污水中氮磷的吸附特性 研究. 应用化工, 2016, 45(9): 1619-1623.]

[22] Fu HM, Jia LM. Study progress of nitrogen and phosphate adsorption \& desorption in soils. Chinese Agricultural Science Bulletin, 2009, 25(21): 198-203. [付海曼, 贾黎明. 土壤对氮、磷吸附/解吸附特性研究进展. 中国农学通报, 
2009, 25(21): 198-203.]

[23] Wang SR, Jin XC, Zhao HC et al. Effect of DOM on phosphate sorption in lake sediments. Acta Pedologica Sinica, 2005, 42(5) : 805-811. [王圣瑞, 金相灿, 赵海超等. 湖泊沉积物中水溶性有机质对吸附磷的影响. 土壤学报, 2005, 42 (5): 805-811.]

[24] Li BG, Qiao YB, Ma Q. Adsorption and desorption of phosphate onto surface sediments from the Yellow River. Journal of Inner Mongolia Normal University: Natural Science Edition, 2010, 39(1) : 50-54, 58. [李北罡, 乔亚斌, 马钦. 黄河表 层沉积物对磷的吸附与释放研究. 内蒙古师范大学学报：自然科学汉文版, 2010, 39(1): 50-54, 58.]

[25] Ji XC, Li HB, Yu YY et al. Ammonia and nitrogen adsorption behavior of ditch sediment after paddy field water-break in estuary. Chinese Agricultural Science Bulletin, 2015, 31(32): 148-153. [季现超, 李海波, 余艳艳等. 河口区稻田退 水后沟渠底泥氨氮的吸附行为. 中国农学通报, 2015, 31(32): 148-153.]

[26] Bhatti JS, Comerford NB, Johnston CT. Influence of oxalate and soil organic matter on sorption and desorption of phosphate onto a spodic horizon. Soil Science Society of America Journal, 1998, 62(4) : 1089-1095.

[27] Rozic M, Cerjan-Stefanovic S, Kurajica S et al. Ammoniacal nitrogen removal from water by treatment with clays and zeolites. Water Research, 2000, 34(14) : 3675-3681.

[28] Ozcan AS, Erdem B, Ozcan A. Adsorption of Acid Blue 193 from aqueous solutions onto Na-bentonite and DTMA-bentonite. Journal of Colloid and Interface Science, 2004, 280(1) : 44-54.

[29] Wang LX, Lv JL, Zhuang XY et al. Characteristics of phosphorus adsorption on paddy soil and river sediment in East China. Soils, 2009, 41(3) : 402-407. [王丽香, 吕家珑, 庄舜尧等. 我国东部河网地区土壤与河道底泥对磷的吸附特 性比较研究. 土壤, 2009, 41(3): 402-407.]

[30] Wang EL, Wang SQ. Adsorption characteristics of phosphorus from different grain sizes sediment in western Liao River. China Environmental Science, 2012, 32(6) : 1054-1061. [王而力, 王嗣淇. 西辽河不同粒级沉积物对磷的吸附特征. 中国环境科学, 2012, 32(6): 1054-1061.]

[31] Wang EL, Wang YD, Wang SQ. Sorption and desorption of ammonium nitrogen on sediments of different grain sizes in western Liao River. Research of Environmental Sciences, 2012, 25(9) : 1016-1023. [王而力, 王雅迪, 王嗣淇. 西辽河 不同粒级沉积物的氨氮吸附-解吸特征. 环境科学研究, 2012, 25(9): 1016-1023. ]

[32] Li BG, Gao N, Ma Q. Adsorption and release behaviors of phosphate on sediments in the upper and middle reaches of Yellow River. Journal of Ecology and Rural Environment, 2010, 26(4) : 356-360. [李北罡, 高娜, 马钦. 黄河上中游 水体沉积物对磷酸盐的吸附/释放行为. 生态与农村环境学报, 2010, 26(4) : 356-360.]

[33] Zhao HY, Wang GP, Liu JS et al. Phosphorus sorption/desorption characteristics of wetland soils in Sanjiang Plain. Ecology and Environment, 2006, 15(5): 930-935. [赵海洋, 王国平, 刘景双等. 三江平原湿地土壤磷的吸附与解吸研 究. 生态环境, 2006, 15(5): 930-935.]

[34] Chen JP, Bai Y, Zhang HJ et al. Ammonia adsorption/desorption behavior in paddy soil from lower position of Liao River plain. Journal of Liaoning Technical University: Natural Science, 2014, 33(1): 94-98. [陈建平, 白杨, 张会杰等. 下 辽河平原水稻田土中氨氮吸附/解吸动力学. 辽宁工程技术大学学报: 自然科学版, 2014, 33(1): 94-98.]

[35] Xu MD, Wei HP, Li M et al. A study on the sorption and desorption of phosphate by sediments in the Yangtze River Estuary. Journal of Taiyuan University of Technology, 2006, 37 (1) : 48-50, 54. [徐明德, 韦鹤平, 李敏等. 长江口泥沙与 沉积物对磷酸盐的吸附和解吸研究. 太原理工大学学报, 2006, 37 (1) : 48-50, 54.]

[36] Chen LL, Zhang CJ, Li P et al. Study on ammonia nitrogen adsorption characteristics of different substrates in constructed wetland. Ecology and Environmental Sciences, 2012, 21(3) : 518-523. [陈丽丽, 张成军, 李鹏等. 人工湿地不同基质 对氨氮的吸附特性研究. 生态环境学报, 2012, 21(3): 518-523.]

[37] Zhang DS, Zhang JF, Shen B. Quasi-analytical solution for advection-dispersion model of solute transport through soils under steady state flow. Journal of Northwest A \& F University: Natural Science Edition, 2009, 37(3) : 187-192. [张德生, 张建丰, 沈冰. 考虑吸附和降解时溶质在土壤中运移的对流-弥散模型及其准解析解. 西北农林科技大学学报: 自 然科学版, 2009, 37(3): 187-192.] 
附表 1 氨氮典型断面各深度沉积物上吸附过程的模型拟合结果

Appendix 1 Simulation result of ammonia nitrogen adsorption at each depth sediments of typical sections

\begin{tabular}{|c|c|c|c|c|c|c|c|c|c|c|}
\hline \multirow{3}{*}{ 动力学模型 } & \multirow{3}{*}{ 参数 } & \multicolumn{9}{|c|}{ 断面 } \\
\hline & & \multicolumn{3}{|c|}{ 土沟 } & \multicolumn{3}{|c|}{ 榆林庄 } & \multicolumn{3}{|c|}{ 和合站 } \\
\hline & & $0 \sim 20 \mathrm{~cm}$ & $20 \sim 40 \mathrm{~cm}$ & $40 \sim 60 \mathrm{~cm}$ & $0 \sim 20 \mathrm{~cm}$ & $20 \sim 40 \mathrm{~cm}$ & $40 \sim 60 \mathrm{~cm}$ & $0 \sim 20 \mathrm{~cm}$ & $20 \sim 40 \mathrm{~cm}$ & $40 \sim 60 \mathrm{~cm}$ \\
\hline \multirow[t]{5}{*}{ ExpAssoc } & $A_{1}$ & 161.85 & 108.15 & 92.35 & 61.73 & 176.95 & 122.00 & 148.22 & 33.29 & 27.82 \\
\hline & $a_{3}$ & 2.54 & 14.99 & 0.05 & 3.14 & 0.06 & 0.03 & 0.04 & 0.95 & 2.02 \\
\hline & $A_{2}$ & 114.31 & 127.78 & 133.39 & 300.65 & 61.88 & 161.24 & 88.42 & 126.04 & 120.70 \\
\hline & $b_{2}$ & 0.01 & 0.05 & 9.78 & 0.17 & 6.16 & 5.82 & 3.60 & 0.05 & 0.07 \\
\hline & $R^{2}$ & 0.95 & 0.95 & 0.96 & 0.96 & 0.92 & 0.90 & 0.98 & 0.98 & 0.99 \\
\hline Lagergren & $k_{1}$ & 0.17 & 0.16 & 19.63 & 0.12 & 0.24 & 0.19 & 0.14 & 0.12 & 0.21 \\
\hline \multirow[t]{2}{*}{ 一级动力学 } & $q_{\mathrm{e}}$ & 119.18 & 99.82 & 744.22 & 87.82 & 82.13 & 139.83 & 83.31 & 18.66 & 30.22 \\
\hline & $R^{2}$ & 0.79 & 0.93 & 0.79 & 0.53 & 0.91 & 0.65 & 0.87 & 0.36 & 0.64 \\
\hline Lagergren & $k_{2}$ & 0.0088 & 0.0082 & 0.0050 & 0.0259 & 0.0160 & 0.0052 & 0.0150 & 0.1718 & 0.0566 \\
\hline \multirow[t]{2}{*}{ 二级动力学 } & $q_{\mathrm{e}}$ & 277.78 & 211.00 & 217.50 & 357.70 & 238.70 & 278.03 & 237.60 & 159.00 & 150.00 \\
\hline & $R^{2}$ & 0.99 & 0.99 & 0.99 & 1.00 & 1.00 & 0.99 & 1.00 & 1.00 & 1.00 \\
\hline \multirow{3}{*}{$\begin{array}{c}\text { Weber-Morris } \\
\text { 扩散 }\end{array}$} & $k_{3}$ & 119.38 & 98.79 & 69.45 & 206.16 & 142.82 & 104.28 & 131.43 & 117.68 & 101.16 \\
\hline & $a_{1}$ & 40.39 & 25.61 & 31.89 & 42.21 & 24.63 & 40.68 & 27.22 & 12.76 & 14.07 \\
\hline & $R^{2}$ & 0.79 & 0.66 & 0.89 & 0.43 & 0.45 & 0.82 & 0.67 & 0.33 & 0.36 \\
\hline \multirow[t]{3}{*}{ Elovich } & $a_{2}$ & 182.95 & 139.17 & 119.38 & 278.52 & 182.08 & 168.03 & 174.53 & 138.25 & 123.78 \\
\hline & $b_{1}$ & 30.56 & 19.93 & 22.20 & 43.56 & 22.49 & 28.60 & 22.63 & 13.44 & 14.35 \\
\hline & $R^{2}$ & 0.93 & 0.81 & 0.88 & 0.82 & 0.76 & 0.82 & 0.95 & 0.75 & 0.75 \\
\hline
\end{tabular}

附表 2 磷酸盐在典型断面各深度沉积物上吸附过程的模型拟合结果

Appendix 2 Simulation result of phosphorus adsorption at each depth sediments of typical sections

\begin{tabular}{|c|c|c|c|c|c|c|c|c|c|c|}
\hline \multirow{3}{*}{ 动力学模型 } & \multirow{3}{*}{ 参数 } & \multicolumn{9}{|c|}{ 断面 } \\
\hline & & \multicolumn{3}{|c|}{ 土沟 } & \multicolumn{3}{|c|}{ 榆林庄 } & \multicolumn{3}{|c|}{ 和合站 } \\
\hline & & $0 \sim 20 \mathrm{~cm}$ & $20 \sim 40 \mathrm{~cm}$ & $40 \sim 60 \mathrm{~cm}$ & $0 \sim 20 \mathrm{~cm}$ & $20 \sim 40 \mathrm{~cm}$ & $40 \sim 60 \mathrm{~cm}$ & $0 \sim 20 \mathrm{~cm}$ & $20 \sim 40 \mathrm{~cm}$ & $40 \sim 60 \mathrm{~cm}$ \\
\hline \multirow[t]{5}{*}{ ExpAssoc } & $A_{1}$ & 672.23 & 371.05 & 357.66 & 464.72 & 267.78 & 180.97 & 121.35 & 87.15 & 145.26 \\
\hline & $a_{3}$ & 4.11 & 6.58 & 7.53 & 5.95 & 0.01 & 0.01 & 0.02 & $2.30 \times 10^{-5}$ & 4.47 \\
\hline & $A_{2}$ & 156.68 & 212.66 & 129.21 & 477.80 & 348.42 & 516.99 & 281.93 & 190.40 & 127.26 \\
\hline & $b_{2}$ & 0.02 & 0.01 & 0.01 & 0.0002 & 3.86 & 4.93 & 10.30 & 2.50 & 0.0002 \\
\hline & $R^{2}$ & 0.98 & 0.99 & 0.99 & 0.87 & 0.95 & 0.94 & 0.97 & 0.99 & 0.98 \\
\hline Lagergren & $k_{1}$ & 0.12 & 0.15 & 0.18 & 0.26 & 0.17 & 0.14 & 0.15 & 0.44 & 0.14 \\
\hline \multirow[t]{2}{*}{ 一级动力学 } & $\mathrm{q}_{e}$ & 689.60 & 382.38 & 370.34 & 453.31 & 338.22 & 483.17 & 265.16 & 195.75 & 134.49 \\
\hline & $R^{2}$ & 0.98 & 0.99 & 0.99 & 0.96 & 0.96 & 0.92 & 0.97 & 0.99 & 0.92 \\
\hline Lagergren & $k_{2}$ & 0.0008 & 0.0018 & 0.0016 & 0.0020 & 0.0026 & 0.0012 & 0.0021 & 0.0068 & 0.0065 \\
\hline \multirow[t]{2}{*}{ 二级动力学 } & $q_{\mathrm{e}}$ & 909.09 & 588.24 & 476.19 & 909.09 & 625.00 & 714.29 & 370.37 & 277.78 & 277.78 \\
\hline & $R^{2}$ & 0.99 & 0.99 & 0.98 & 0.99 & 0.99 & 0.98 & 0.97 & 0.99 & 0.99 \\
\hline Weber-Morris & $k_{3}$ & 136.03 & 182.53 & 98.58 & 454.90 & 251.33 & 161.96 & 92.25 & 99.85 & 124.04 \\
\hline \multirow[t]{2}{*}{ 扩散 } & $a_{1}$ & 167.42 & 88.16 & 82.28 & 107.84 & 90.36 & 123.45 & 60.16 & 45.39 & 34.90 \\
\hline & $R^{2}$ & 0.95 & 0.98 & 0.99 & 0.87 & 0.92 & 0.91 & 0.98 & 0.84 & 0.94 \\
\hline \multirow[t]{3}{*}{ Elovich } & $a_{2}$ & 397.98 & 320.20 & 226.94 & 623.32 & 393.08 & 354.71 & 186.08 & 171.07 & 178.60 \\
\hline & $b_{1}$ & 114.42 & 58.20 & 53.29 & 71.39 & 64.65 & 81.33 & 38.79 & 32.69 & 23.54 \\
\hline & $R^{2}$ & 0.90 & 0.87 & 0.84 & 0.78 & 0.96 & 0.80 & 0.83 & 0.89 & 0.87 \\
\hline
\end{tabular}


附表 3 氨氮在典型断面各深度沉积物上解吸过程的模型拟合结果

Appendix 3 Simulation result of ammonia nitrogen desorption at each depth sediments of typical sections

\begin{tabular}{|c|c|c|c|c|c|c|c|c|c|c|}
\hline \multirow{3}{*}{ 动力学模型 } & \multirow{3}{*}{ 参数 } & \multicolumn{9}{|c|}{ 断面 } \\
\hline & & \multicolumn{3}{|c|}{ 土沟 } & \multicolumn{3}{|c|}{ 榆林庄 } & \multicolumn{3}{|c|}{ 和合站 } \\
\hline & & $0 \sim 20 \mathrm{~cm}$ & $20 \sim 40 \mathrm{~cm}$ & $40 \sim 60 \mathrm{~cm}$ & $0 \sim 20 \mathrm{~cm}$ & $20 \sim 40 \mathrm{~cm}$ & $40 \sim 60 \mathrm{~cm}$ & $0 \sim 20 \mathrm{~cm}$ & $20 \sim 40 \mathrm{~cm}$ & $40 \sim 60 \mathrm{~cm}$ \\
\hline \multirow[t]{5}{*}{ ExpAssoc } & $A_{1}$ & 91.81 & 105.72 & 67.63 & 98.78 & 33.59 & 76.77 & 73.82 & 76.85 & 24.73 \\
\hline & $a_{3}$ & 0.01 & 0.06 & $10 \times 10^{-7}$ & 0.62 & 1.36 & $3 \times 10^{-4}$ & 0.15 & $1.3 \times 10^{-4}$ & 42.87 \\
\hline & $A_{2}$ & 108.96 & 37.41 & 45.50 & 76.67 & 96.52 & 66.59 & 57.20 & 48.37 & 90.33 \\
\hline & $b_{2}$ & 2.82 & 6.42 & 0.96 & $2 \times 10^{-3}$ & $6 \times 10^{-4}$ & 2.05 & $2 \times 10^{-3}$ & 6.55 & $1.6 \times 10^{-4}$ \\
\hline & $R^{2}$ & 0.98 & 0.97 & 0.80 & 0.84 & 0.93 & 0.99 & 0.77 & 0.97 & 0.60 \\
\hline Lagergren & $k_{1}$ & 0.08 & 0.10 & 0.06 & 0.05 & 0.07 & 0.10 & 0.06 & 0.07 & 0.04 \\
\hline \multirow[t]{2}{*}{ 一级动力学 } & $q_{\mathrm{e}}$ & 72.21 & 39.26 & 29.58 & 58.75 & 20.33 & 30.28 & 23.39 & 38.41 & 15.60 \\
\hline & $R^{2}$ & 0.89 & 0.93 & 0.52 & 0.48 & 0.86 & 0.77 & 0.52 & 0.85 & 0.65 \\
\hline Lagergren & $k_{2}$ & 0.0082 & 0.0159 & 0.0156 & 0.0081 & 0.0342 & 0.0251 & 0.0196 & 0.0139 & 0.0227 \\
\hline \multirow[t]{2}{*}{ 二级动力学 } & $q_{\mathrm{e}}$ & 208.33 & 144.93 & 123.46 & 200.00 & 135.14 & 144.93 & 142.96 & 126.58 & 106.38 \\
\hline & $R^{2}$ & 0.999 & 0.999 & 0.997 & 0.997 & 1.000 & 1.000 & 0.999 & 0.999 & 0.999 \\
\hline Weber-Morris & $k_{3}$ & 112.56 & 100.81 & 84.72 & 122.80 & 107.50 & 94.52 & 112.35 & 78.53 & 88.28 \\
\hline \multirow[t]{2}{*}{ 扩散 } & $a_{1}$ & 17.24 & 7.81 & 6.72 & 13.04 & 4.96 & 9.81 & 5.20 & 8.12 & 2.61 \\
\hline & $R^{2}$ & 0.74 & 0.78 & 0.65 & 0.63 & 0.67 & 0.65 & 0.52 & 0.87 & 0.76 \\
\hline \multirow[t]{3}{*}{ Elovich } & $a_{2}$ & 136.14 & 111.46 & 93.69 & 139.79 & 113.98 & 107.31 & 119.07 & 90.39 & 92.23 \\
\hline & $b_{1}$ & 20.17 & 9.16 & 8.14 & 16.34 & 6.19 & 12.27 & 6.60 & 8.54 & 2.56 \\
\hline & $R^{2}$ & 0.93 & 0.99 & 0.88 & 0.90 & 0.95 & 0.94 & 0.76 & 0.88 & 0.67 \\
\hline
\end{tabular}

附表 4 磷酸盐在典型断面各深度沉积物上解吸过程的模型拟合结果

Appendix 4 Simulation result of phosphorus desorption at each depth sediments of typical sections

\begin{tabular}{|c|c|c|c|c|c|c|c|c|c|c|}
\hline \multirow{3}{*}{ 动力学模型 } & \multirow{3}{*}{ 参数 } & \multicolumn{9}{|c|}{ 断面 } \\
\hline & & \multicolumn{3}{|c|}{ 土沟 } & \multicolumn{3}{|c|}{ 榆林庄 } & \multicolumn{3}{|c|}{ 和合站 } \\
\hline & & $0 \sim 20 \mathrm{~cm}$ & $20 \sim 40 \mathrm{~cm}$ & $40 \sim 60 \mathrm{~cm}$ & $0 \sim 20 \mathrm{~cm}$ & $20 \sim 40 \mathrm{~cm}$ & $40 \sim 60 \mathrm{~cm}$ & $0 \sim 20 \mathrm{~cm}$ & $20 \sim 40 \mathrm{~cm}$ & $40 \sim 60 \mathrm{~cm}$ \\
\hline \multirow[t]{5}{*}{ ExpAssoc } & $A_{1}$ & 13.27 & 43.21 & 66.70 & 28.11 & 36.96 & 19.50 & 79.09 & 49.08 & 30.74 \\
\hline & $a_{3}$ & 0.10 & 4.91 & 0.05 & 3.29 & 0.09 & 2.14 & $9.5 \times 10^{-5}$ & 1.92 & 1.92 \\
\hline & $A_{2}$ & 45.50 & 52.44 & 19.51 & 24.74 & 13.93 & 18.17 & 53.31 & 57.83 & 64.54 \\
\hline & $b_{2}$ & 2.53 & $1.2 \times 10^{-3}$ & 3.97 & $1.6 \times 10^{-3}$ & 24.50 & $1.7 \times 10^{-3}$ & 4.07 & $1.4 \times 10^{-3}$ & $8.4 \times 10^{-4}$ \\
\hline & $R^{2}$ & 0.98 & 0.89 & 0.93 & 0.93 & 0.88 & 0.79 & 0.94 & 0.84 & 0.85 \\
\hline Lagergren & $k_{1}$ & 0.08 & 0.08 & 0.09 & 0.09 & 0.05 & 0.09 & 0.10 & 0.09 & 0.10 \\
\hline \multirow[t]{2}{*}{ 一级动力学 } & $q_{\mathrm{e}}$ & 28.64 & 33.20 & 19.40 & 20.55 & 14.09 & 13.74 & 35.55 & 42.57 & 16.58 \\
\hline & $R^{2}$ & 0.85 & 0.88 & 0.78 & 0.92 & 0.61 & 0.87 & 0.87 & 0.96 & 0.64 \\
\hline Lagergren & $k_{2}$ & 0.017 & 0.016 & 0.035 & 0.028 & 0.030 & 0.041 & 0.018 & 0.012 & 0.040 \\
\hline \multirow[t]{2}{*}{ 二级动力学 } & $q_{\mathrm{e}}$ & 61.73 & 98.04 & 88.50 & 54.95 & 49.02 & 40.32 & 133.33 & 117.65 & 98.04 \\
\hline & $R^{2}$ & 0.999 & 0.999 & 0.999 & 0.999 & 0.996 & 0.999 & 0.999 & 0.998 & 0.999 \\
\hline Weber-Morris & $k_{3}$ & 21.24 & 55.76 & 64.85 & 28.95 & 31.51 & 22.51 & 85.45 & 69.11 & 72.49 \\
\hline \multirow[t]{2}{*}{ 扩散 } & $a_{1}$ & 7.48 & 7.33 & 4.15 & 4.65 & 2.88 & 3.19 & 8.73 & 8.23 & 4.77 \\
\hline & $R^{2}$ & 0.69 & 0.81 & 0.70 & 0.78 & 0.72 & 0.70 & 0.78 & 0.77 & 0.66 \\
\hline \multirow[t]{3}{*}{ Elovich } & $a_{2}$ & 31.16 & 65.98 & 70.88 & 35.32 & 35.41 & 26.77 & 97.54 & 80.35 & 78.76 \\
\hline & $b_{1}$ & 9.15 & 8.28 & 4.96 & 5.41 & 3.42 & 3.86 & 10.02 & 9.65 & 5.91 \\
\hline & $R^{2}$ & 0.95 & 0.95 & 0.93 & 0.97 & 0.93 & 0.94 & 0.94 & 0.97 & 0.93 \\
\hline
\end{tabular}

